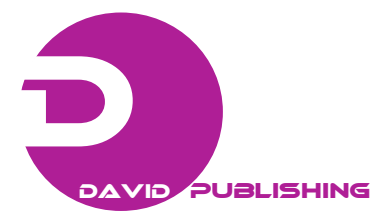

\title{
The Effects of Touch Button Size on Touchscreen
}

\section{Operability}

\author{
Jinghong Xiong ${ }^{1}$, Satoshi Muraki ${ }^{2}$ and Kiyotaka Fukumoto ${ }^{3}$ \\ 1. Graduate School of Design, Kyushu University, Fukuoka 815-8540, Japan \\ 2. Faculty of Design, Kyushu University, Fukuoka 815-8540, Japan \\ 3. Faculty of Engineering, Shizuoka University, Shizuoka 432-8561, Japan
}

Received: June 27, 2014 / Accepted: July 11, 2014 / Published: August 25, 2014.

\begin{abstract}
The present study investigated the effects of touch button size on touchscreen operability and compared these effects between young adult and elderly participants. A total of 21 young adults (aged $22.3 \pm 1.5$ years) and 20 elderly adults (aged $68.1 \pm$ 4.9 years) were recruited and asked to press square number buttons (from 0 to 9 ) on an experimental touchscreen with their right index finger. The buttons' size changed during the experiment with six conditions (6, 8, 10, 12, 14 and $16 \mathrm{~mm})$. It was found that a decrease of the button size to $10 \mathrm{~mm}$ or below tended to increase the operation time and error rate, whereas it decreased the subjective overall operability of the touchscreen. Such effects were greater in the elderly adults than in the young adults. In addition, the reaction positions on the buttons were found to be close to the right side of them, which led the fingertip to approach the right outline of the buttons. These findings suggest that the use of small touch buttons should be minimised on touchscreens, especially for elderly users.
\end{abstract}

Key words: Touchscreen, button size, operability, elderly.

\section{Introduction}

In recent years, touchscreen technology has been developing rapidly. Many of the electrical devices that people use in their everyday lives come with touchscreens, as well as touch buttons on the screens. Differing from the fixed push buttons in tactile keypads, designers have more freedom to change the size, shape, position and arrangement of touch buttons. Small buttons, in particular, used frequently in handheld touchscreen devices, such as smartphones, tablet PCs, pocket music players and driving navigators. The advantage of using small buttons is that even a small handheld touchscreen can display many buttons, so we can reduce the time that users spend flipping through menus in order to search the appropriate buttons. However, correctly selecting and

Corresponding author: Jinghong Xiong, Ph.D., research fields: ergonomics, human engineering and design for better use. E-mail: jimkuma@design.kyushu-u.ac.jp. pressing a small button also requires acceptable motor skills in the operating fingers. Otherwise, input errors from pressing incorrect buttons, and the time and physical efforts spent in correcting these would be increased. As a result, the user experience will be negatively affected [1-3]. This means that small touch buttons have great potential to affect the operability of touchscreens.

On the other hand, age could also be another factor affecting the operability of touchscreen devices. In recent decades, there has been an ongoing increase in the aging population all over the world, especially in developed countries. It was found that elderly users tend to be slower and make more input errors than young adults when operating a touch panel interface [4-6]. It was asserted that elderly users could use push buttons instead, since they are preferable for them. However, the reality is that touchscreen devices have been taking an increasingly large market share 
globally. Thus, elderly users may have limited choices of push button devices, and they have to use touchscreens more. Against this background, ergonomics designers should be reminded that the size of touchscreens is a major design issue, especially in order to improve the operability of touchscreens for elderly users.

This study aimed to shed light on the effects of touch button size (6 $\mathrm{mm}$ to $16 \mathrm{~mm}$ ) to the operability of a touchscreen, and to compare the effects between young adults and elderly users. In addition, this study also investigated the reasons for the increased input errors with increasing button size, from the perspective of the reaction position between the index fingertip and the touchscreen surface.

The paper is organized as follows: Section 2 introduces the study methods; Section 3 presents the experimental results; Section 4 is the discussion of the results; and Section 5 concludes the study by providing suggestions for the designs of touchscreen button size.

\section{Methods}

A total of 21 young adults (10 males, 11 females, aged $22.3 \pm 1.5$ years) and 20 elderly citizens (10 males, 10 females, aged $68.1 \pm 4.9$ years) were recruited. The body heights and widths of the distal interphalangeal joint of the index finger were $165.1 \pm$ $6.2 \mathrm{~cm}$ and $160.3 \pm 4.7 \mathrm{~cm}$, and $15.3 \pm 1.2 \mathrm{~mm}$ and $16.3 \pm 1.4 \mathrm{~mm}$, respectively, in the young and elderly participants. All of them were right-handed and had sufficient vision to recognize the buttons and the outlines of the buttons displayed on the experimental touchscreen. This study was approved by the Institutional Ethics Review Board of Kyushu University, Japan, and all participants agreed to take part in the experiment.

An original program based on Visual Basic 6.0 that displays the input numbers and manages the interface (length $158.0 \mathrm{~mm} \times$ width $68.0 \mathrm{~mm}$ ) in the experimental touchscreen device was developed for the study.

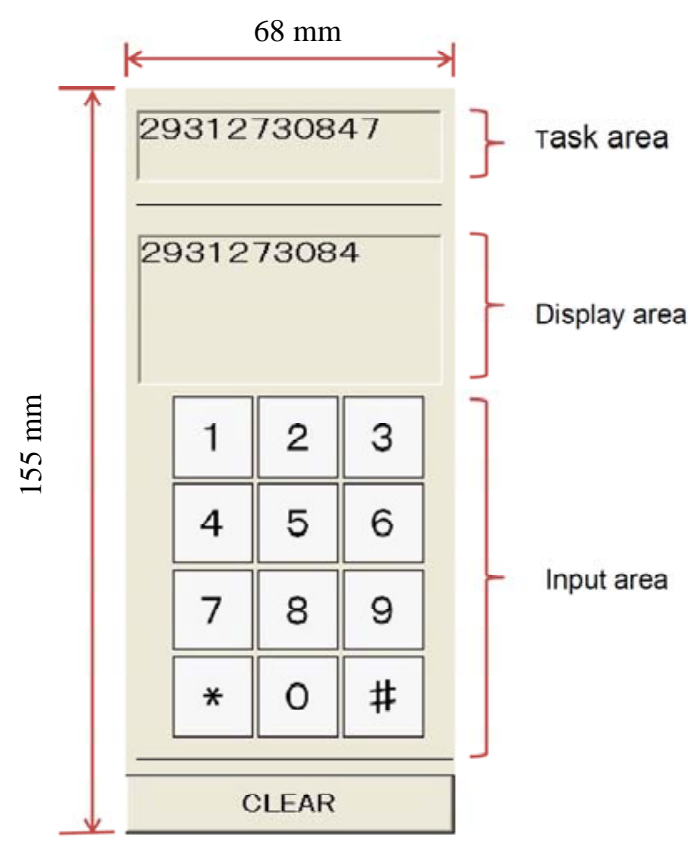

Fig. 1 Experimental interface.

The experimental interface was at the centre of the touchscreen, which consisted of three areas, namely, task area, display area and input area (Fig. 1). The task area was at the upper part of the screen showing the text $(0$ to $9, *, \#)$ that the participants were asked to input. The input area was at the bottom of the screen where 12 square buttons were arranged as a matrix of three rows and four columns. The text ( 0 to $9, *$, \#) was displayed at the centre of each button. The size of each button among six different size conditions $(6,8$, 10, 12, 14 and $16 \mathrm{~mm}$ ) changes. These 12 buttons were displayed at the centre of the input area with an interval of $1 \mathrm{~mm}$ between them. Thus, the display area was between the task and input areas that displayed the texts that the participants input in real time.

All participants sat comfortably on an armless chair in front of a desk with a touchscreen device, and they were asked to perform the tasks with their right index finger (Fig. 2). The task area displayed the text consisting of an 11-digit number (including * and \#) selected randomly. When the text was displayed on the task area, the participants were asked to input text matching it immediately. Meanwhile, after each button was tapped using the index finger, the number 


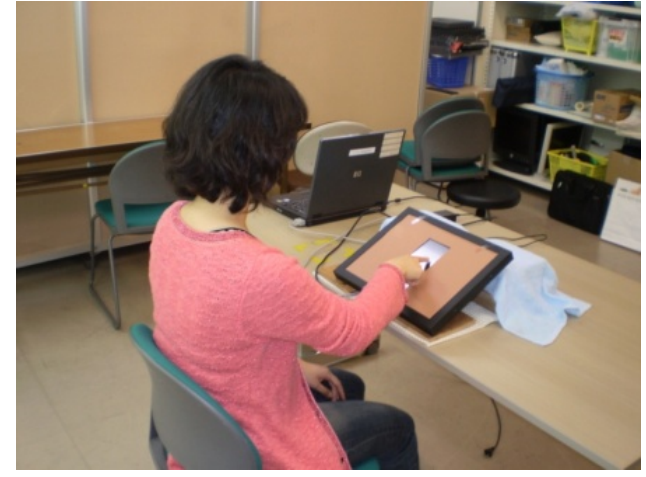

Fig. 2 Operating posture in experiment.

corresponding to the button was displayed in the display area immediately without a sound or any other visual feedback occurring. The participants were instructed to perform the task as rapidly and accurately as possible. Five trials were applied for each button size condition, and the order of button sizes was counter-balanced.

The program can detect the timing at which the fingertip moves away from the surface of the screen. Thus, the interval from the time when the fingertip moves away from a button to the time of moving away from the next button, is defined as the operation time in this study.

The number of errors that the participants made in each trial is defined as the error rate in this study. The errors included not touching the targeted buttons, unnecessary touching of the targeted buttons, and the reaction position being outside the outline of the targeted button.

The participants were asked to evaluate subjectively the overall operability of each button size. They marked this using a scale from -50 to +50 , where -50 stands for the most negative score and +50 stands for the most positive one.

The position at which the fingertip left the screen surface could also be recorded using the program. This position is defined as the reaction position in this study.

The results obtained from the measurements were analysed by repeated two-way analysis of variance (age group $\times$ button size). In addition, Bonferroni test was applied for comparison among the six button size conditions when a significant effect of button size was found in the repeated two-way analysis of variance. Statistical significance was accepted at a $p$-value of less than 0.05 .

\section{Results}

The operation time revealed significant effects of age and button size, as well as the interaction of these two factors (Fig. 3). The elderly participants showed a longer operation time than the young adults at all button sizes by roughly twofold. Even though the operation time increased with decreasing button size in all participants, the effects of button size were significantly greater in the elderly than in the young.

The error rate also showed a significant increase when the button size was reduced to $10 \mathrm{~mm}$ or below in both age groups (Fig. 4). However, this effect became much greater, when the button size was reduced to $6 \mathrm{~mm}$, especially in the elderly group, despite minor differences between the age groups at the button sizes of 12, 14 and $16 \mathrm{~mm}$.

In terms of subjective overall operability, a main effect was found only in button size. The rating score decreased with decreasing button size in both age groups (Fig. 5). The mean of the score even dropped below 0 when the button size was reduced to $6 \mathrm{~mm}$. In

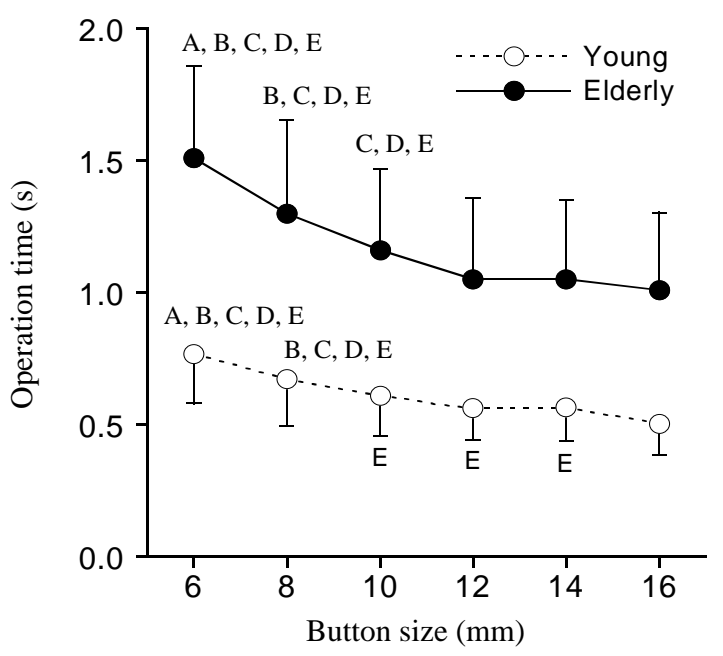

Fig. 3 Operation time at each button size in young and elderly groups. A, B, C, D and E: $p<0.05$ vs. 8, 10, 12, 14 and $16 \mathrm{~mm}$. 


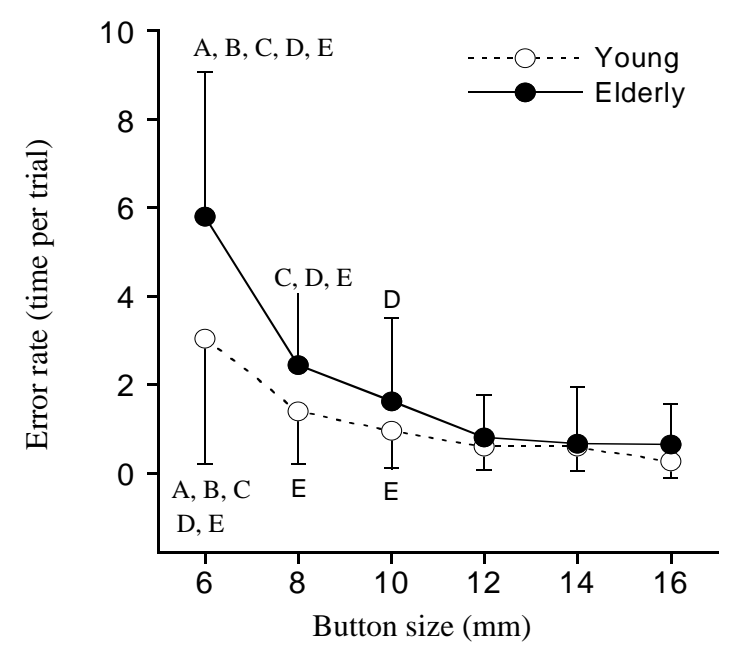

Fig. 4 Error rate at each button size in young and elderly groups. A, B, C, D and E: $p<0.05$ vs. 8, 10, 12, 14 and 16 mm.

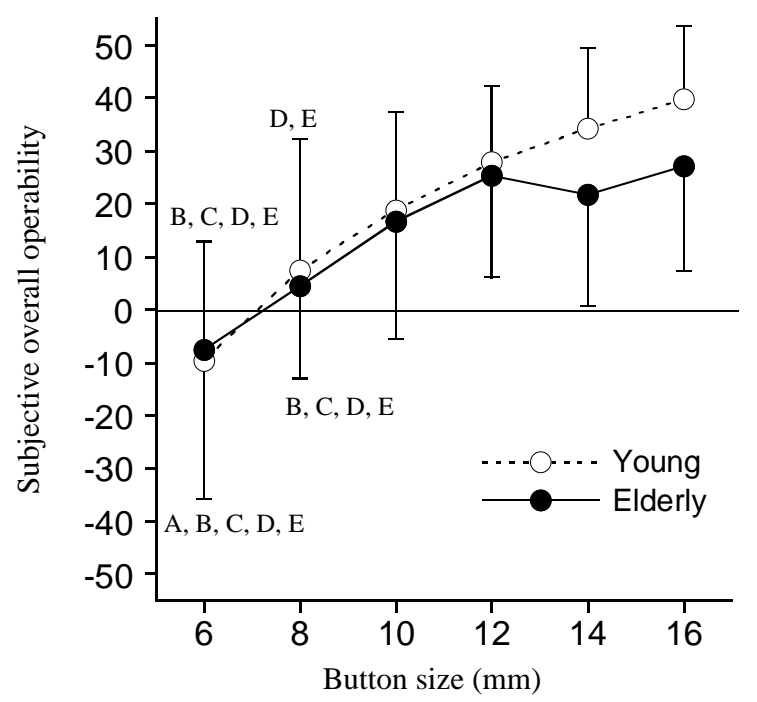

Fig. 5 Subjective operability in young and elderly groups. A, B, C, D and E: $p<0.05$ vs. 8, 10, 12, 14 and $16 \mathrm{~mm}$.

the elderly group, the scores remained unchanged at button sizes between $12 \mathrm{~mm}$ and $16 \mathrm{~mm}$.

Significant differences of reaction position were found between the age groups. Fig. 6 shows red (black if colours cannot display) dots that represent the mean reaction positions (two-dimensional) on all touch buttons ( 0 to 9 ) for each button size in the young and elderly groups. The gaps from the button centre were greater in the horizontal direction than in the vertical direction in both groups. This means that the reaction positions tended to be placed to the right side of the

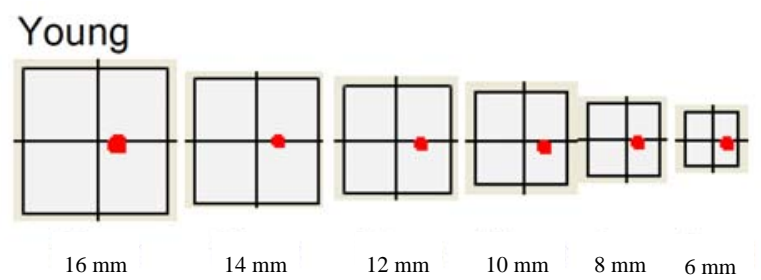

Elderly

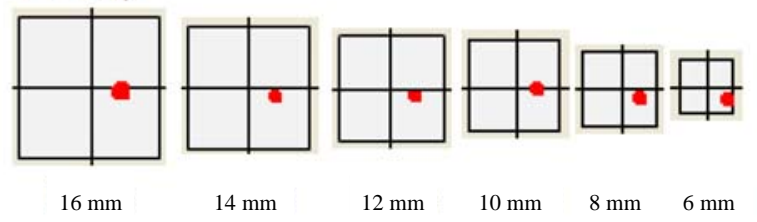

Fig. 6 Reaction position with each button size condition in young and elderly groups.

button regardless of the button size. In addition, the gaps were particularly large from the button centre in the elderly group, which also indicates that the reaction position of elderly participants approached the right edge of the button.

\section{Discussions}

This study found that touch button size significantly affects the operability of touchscreens. The operation time, error rate and subjective overall operability significantly varied in both young and elderly participants with decreasing button size. Especially when the button size was reduced to $6 \mathrm{~mm}$, all of the measures showed that the human performance in both age groups was significantly reduced. In addition, this effect of touchscreen operability was greater in elderly participants than in young ones. It is considered that elderly participants generally have decreased motor functions, muscle strength and visual abilities [7-9], so when the touch buttons are reduced to an extremely small size, such as $6 \mathrm{~mm}$, the difficulty for the users in recognising the buttons is also greatly increased. Thus, it is suggested that a touch button size of $6 \mathrm{~mm}$ or below should be avoided in touchscreen devices for elderly users.

On the other hand, this study also indicates that increasing the button size to $12 \mathrm{~mm}$ or above does not necessarily improve the operability of touchscreens. In terms of the error rate and subjective overall 
operability, no significant difference was found among the button sizes between $12 \mathrm{~mm}$ and $16 \mathrm{~mm}$. However, the overall operability decreased at a button size of $10 \mathrm{~mm}$ or below. A previous study proposed that a larger button size on touchscreens could improve user performance. However, larger buttons tend to take up more space on the screen, and the number of buttons that can be displayed is limited as a result. In addition, the number can be even more limited by the small size of touchscreens. This implies that users would have to flip through more menus to find the appropriate button. Consequently, human performance and user experience are likely to be reduced. Thus, in cases of a small touch panel for which a fair amount of inputting is needed, a button size of 10-12 mm would give an acceptable balance between ease of operation and the number of buttons displayed on a touchscreen.

Under the present experimental conditions, it was found that the reaction positions were shifted to the right side from the button centre in all of the participants. This means that the participants pressed the right side of the buttons rather than their centre. It is considered that this occurred because a particular side of the operating index fingertip was used, which led the finger to approach the right side of the buttons. During the experiment, all participants were asked to perform the task with their right index fingers by pressing a button and then immediately releasing the finger from the surface of the touchscreen. Instantly, after the fingertip left the surface of the touchscreen, the position of the pressing movement was recorded by the system as the reaction position. This system has been widely used in small touchscreen devices, such as smartphones and tablets; thus, the results obtained by this system are reliable to represent the actual positions at which the finger left the touchscreen surface. The significance of this is that, according to the reaction position, the index finger was shifted to the right side of the targeted buttons during the entire pressing action from beginning to end. This could have resulted in a particular part of the index fingertip being used. Thus, at the instant when the finger left the screen surface, the position pressed by the fingertip was shown to be at the right side of the target buttons. In addition, button size and age did not show any significant effects on the reaction position, even though they could have affected the operation time, error rate and overall operability in this study. These results suggest that there are some gaps between the perceived and actual reaction positions in the participants. This could be a potential problem for the operability of touchscreens, as well as a specific issue in the design of touchscreen interfaces.

Furthermore, no significant main effect of button size was identified for the horizontal distance from the button centre to the reaction position. However, it was found that a smaller button size tended to induce the reaction position to be closer to the outline of the buttons. At a button size of $6 \mathrm{~mm}$ in the elderly group, the reaction positions were closer to the right outline of the button than in the young group. As stated above, elderly participants tend to have declined motor functions, muscle strength and visual abilities, which could be among the main reasons for their markedly increased error rate at a button size of $6 \mathrm{~mm}$.

The present study may also have limitations. Insufficient experience using touchscreen devices in the elderly participants is considered to be a major limitation in the experiment. Owing to this, their index finger movements may have been somewhat distorted. This could have altered the results in the measurement to a certain degree.

\section{Conclusions}

Overall, three significant suggestions for the design of touchscreen interfaces could be drawn from the above findings. Firstly, smaller touch buttons (a size of $6 \times 6 \mathrm{~mm}^{2}$ or smaller) significantly reduce the operability of a touchscreen, especially in elderly users. Secondly, increasing the button size to $12 \times 12 \mathrm{~mm}^{2}$ or above barely improved the operability of a touchscreen. 
Thirdly, reducing the gaps between the perceived and actual reaction positions at touch buttons tends to improve the overall operability of a touchscreen.

\section{References}

[1] Colle, H., and Hiszem, K. 2004. "Standing at a Kiosk: Effects of Key Size and Spacing on Touch Screen Numeric Keypad Performance and User Preference.” Ergonomics 47: 1406-23.

[2] Harada, H., Katsuura, T., and Kikuchi, Y. 1996. "Fundamental Study on the Size and Inter-Key Spacing of Numeric Keys for Touch Screen.” Journal of Human Ergology 25: 167-74.

[3] Schedlbauer, M. 2007. "Effects of Key Size and Spacing on the Completion Time and Accuracy of Input Tasks on Soft Keypads Using Trackball and Touch Input.” In Proceeding of the Human Factors and Ergonomics Society Annual Meeting 2007: 429-33.
[4] Kurniawan, S. 2008. "Older People and Mobile Phones: A Multi-method Investigation.” International Journal of Human-Computer Studies 66: 889-901.

[5] Muraki, S., Okabe, K., Abe, T., and Sai, A. 2011. “The Preferable Keypad Layout for Ease Of Pressing Small Cell Phone Keys with the Thumb.” Journal of Human Ergology 39: 133-42.

[6] Plocher, J. Z. X., and Kiff, L. T. 2007. “Touch Screen User Interfaces for Older Adults: Button Size and Spacing." Lecture Notes in Computer Science 4554: 933-41.

[7] Carmeli, E., Patish, H., and Coleman, R. 2003. "The Aging Hand”. Journal of Gerontology 58: 146-52.

[8] Cole, K. J., and Rotella, D. L. 2002. "Old Age Impairs the Use of Arbitrary Visual Cues for Predictive Control of Fingertip Force During Grasp.” Journal of Experimental Brain Research 143: 35-41.

[9] Ranganathan, V. K., Siemionow, V., Sahgal, V., and Yue, G. H. 2001. "Effects of Aging on Hand Function." Journal of American Geriatrics Society 49: 1478-84. 